\title{
Incommensurate Numbers, Continued Fractions, and Fractal Immittances
}

\author{
A. Lakhtakia*, R. Messier*,**, V. V. Varadan*, and V. K. Varadan* \\ The Pennsylvania State University, University Park, PA 16802 (USA) \\ Z. Naturforsch. 43a, 943-955 (1988); received June 10, 1988 \\ Continued fractions have a rich tradition in the theory of numbers; e.g., non-terminating con- \\ tinued fractions represent irrational numbers. It will be shown that a class of continued fractions \\ possess the property of self-referential decomposition, and their interpretation in the form of non- \\ terminating ladder circuits gives rise to fractal immittances with potential analogies to rough \\ surfaces, thin cermet films, as well as to the internal void network structure of thick films.
}

\section{Introduction}

The motivation for this work comes from number theory: the representation of irrational numbers in the Stieltjes continued fraction form, and which has already inspired the development of ladder circuits [1]. Continued fractions have recently been used in understanding fractal quantization of particles in onedimensional potentials with incommensurate periods [2], as well as in two-dimensional electron gases [3]: a particularly appealing and simple interpretation of the relevant Hamiltonian has been given by Chao [4]. Continued fractions have also been applied in examining the frustrated instabilities of active optical resonators [5].

Although continued fractions have seen some use in the characterisation of the rough surfaces of real materials $[6,7]$, they have not been applied yet to the inhomogeneous internal structure of materials. It will be shown that specific examples of the resulting ladder circuits have fractal immittances with potential analogies to both cermet thin films and films with internal void networks; and, hence, to the related film properties.

\footnotetext{
* Department of Engineering Science and Mechanics.

** Materials Research Laboratory.

Reprint requests to Prof. R. Messier, Engineering Science and Mechanics, 265 Materials Research Labroatory, The Pennsylvania State University, University Park, PA 16802, USA
}

\section{Continued Fractions and Quadratic Irrational Numbers}

Any rational or irrational number can be written down in the continued fraction form as [8]

$$
\left\langle a_{0}, a_{1}, a_{2}, a_{3}, \ldots\right\rangle=a_{0}+\frac{1}{a_{1}+\frac{1}{a_{2}+\frac{1}{a_{3}+\ldots}},}
$$

with $a_{0}, a_{1}$, etc. being positive integers [but see Appendix]. The continued fraction is finite in size when it represents any rational number $p / q$, with $a_{0}$ being the integral part of the ratio $p / q$; it turns out that every rational number has exactly two such representations [9]. On the other hand, irrational numbers have continued fraction representations which are infinite in size. Of these irrational numbers, there is a class of quadratic irrational numbers which are solutions of a quadratic equation. A theorem due to Lagrange [10] states that the continued fraction expansion of any quadratic irrational is periodic after a certain state, e.g.,

$$
\begin{aligned}
& \sqrt{15}=\langle 3,1,6,1,6,1,6, \ldots\rangle, \\
& \sqrt{31}=\langle 5,1,1,3,5,3,1,1,10,1,1,3,5,3,1,1 \text {, } \\
& 10,1,1,3,5,3,1,1,10, \ldots>\text {, } \\
& \{24-\sqrt{15}\} / 17=\langle 1,5,2,3,2,3,2,3, \ldots\rangle .
\end{aligned}
$$

It is the representation of quadratic irrational numbers which is of particular interest in the present context. The simplest example is the number

$$
Q(\mu)=\langle 0, \mu, \mu, \mu, \ldots\rangle ; \quad \mu>0
$$


in which we will accept $\mu$ as any rational number; $Q$ can achieve two numerical values via the equation $Q=1 /(\mu+Q)$. The next example is the number

$$
\begin{array}{r}
Q\left(\mu_{1}, \mu_{2}\right)=\left\langle 0, \mu_{1}, \mu_{2}, \mu_{1}, \mu_{2}, \ldots\right\rangle ; \\
\mu_{1}, \mu_{2}>0,
\end{array}
$$

which too can achieve two numerical values via the quadratic equation $\mu_{1} Q^{2}+\mu_{1} \mu_{2} Q-\mu_{2}=0$.

This latter function $Q\left(\mu_{1}, \mu_{2}\right)$ forms the basis of the work reported here. We will remove the restriction that $\mu_{1}$ and $\mu_{2}$ be real positive rational numbers, and let them represent (complex) impedances $Z$ and admittances $Y$; we will also assume [see Appendix] that all continued fractions are either finite in size or they converge. The first fundamental form comes out when we set $\mu_{1}=Y_{b}=1 / Z_{b}$ and $\mu_{2}=Z_{a}=1 / Y_{a}$, so that

$$
Q\left(Y_{b}, Z_{a}\right)=0+\frac{1}{Y_{b}+\frac{1}{Z_{a}+\frac{1}{Y_{b}+\ldots}},}
$$

and $Q\left(Y_{b}, Z_{a}\right)+Z_{a}$ can be interpreted as the impedance of the "series-parallel" circuit shown in Figure $1 \mathrm{a}$. The second fundamental form comes around when $\mu_{1}=Z_{b}$ and $\mu_{2}=Y_{a}$ so that

$$
Q\left(Z_{b}, Y_{a}\right)=0+\frac{1}{Z_{b}+\frac{1}{Y_{a}+\frac{1}{Z_{b}+\ldots}},}
$$

and $Q\left(Z_{b}, Y_{a}\right)+Y_{a}$ can be interpreted as the admittance of the "parallel-series" circuit shown in Figure $1 b$.

From the preceding paragraph, it is immediately obvious that $Q\left(Y_{b}, Z_{a}\right)+Z_{a}$ and $Q\left(Z_{b}, Y_{a}\right)+Y_{a}$ are nothing but the immittances of ladder circuits [1]. Usually, however, ladder circuits are terminated in some prescribed fashion; thus, their immittances are "closer" to rational numbers than to irrational numbers.

\section{Circuits of Infinite Size: Impedances}

There is no restriction on ladder circuits that they be terminated after some number of stages, and we seize upon that idea to generalize $Q\left(Y_{b}, Z_{a}\right)+Z_{a}$. Our basic building blocks are two impedances $Z_{a}=1 / Y_{a}$ and $Z_{b}=1 / Y_{b}$, and the circuit will be constructed in a sequential manner. At the zeroth stage, these imped-

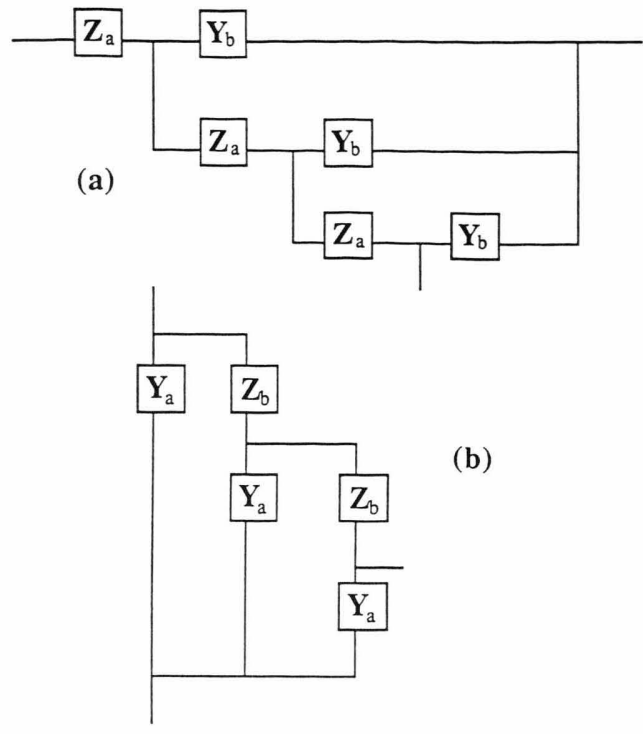

Fig. 1. Ladder circuit representation of (a) $Q\left(Y_{b}, Z_{a}\right)+Z_{a}$ in the "series-parallel" form, and (b) $Q\left(Z_{b}, Y_{a}\right)+Y_{a}$ in the "parallel-series" form.

ances are connected in series, and

$$
Z^{(0)}=Z_{a}+Z_{b} .
$$

Then $Z_{b}$ is shorted by $N_{1}$ parallel branches, each of which carries an impedance $p_{1} Z_{a}$ and $q_{1} Z_{b}$, where $p_{1}$ and $q_{1}$ are appropriate numbers such that $p_{1} Z_{a}$ and $q_{1} Z_{b}$ are realizable. Consequently, the impedance of the circuit at this stage can be represented by

$$
Z^{(1)}=Z_{a}+\frac{1}{Y_{b}+\frac{N_{1}}{p_{1} Z_{a}+q_{1} Z_{b}} .}
$$

In the next stage of growth each of the impedances $q_{1} Z_{b}$ is shorted out by $N_{2}$ parallel branches, each having an impedance $p_{2} Z_{a}+q_{2} Z_{b}$. Thus, the circuit impedance at this stage is given as

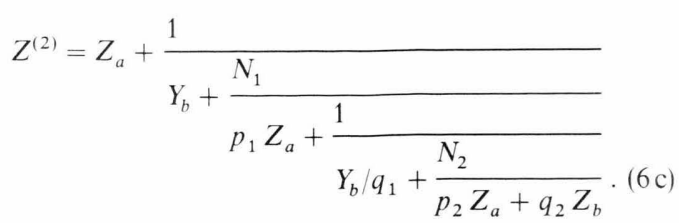

This process continues ad infinitum. Provided the real and the imaginary parts of the sequence $\left\{\boldsymbol{Z}^{(i)}\right\}$ converge, it can be said that

$$
Z^{(x)}=\operatorname{Lim}_{i \rightarrow \infty} Z^{(i)}
$$


will be the impedance of the circuit so obtained. There is a pattern immediately obvious in the development of this circuit. As an example, let $N_{i}=N$ and $p_{i}=q_{i}=a^{i} \forall i \geq 1$. Then, it is easy to verify that

$$
Z^{(i)}=Z_{a}+1 /\left[Y_{b}+(N / a) Y^{(i-1)}\right] ; \quad i>0,
$$

in which $Y^{(i)}=1 / Z^{(i)} \forall i \geq 0$. Provided (6d) holds, then it can be easily seen that $Z^{(\infty)}$ can be obtained as a solution of the quadratic equation

$$
\begin{aligned}
{\left[Z^{(\infty)}\right]^{2} Y_{b} } & +\left[Z^{(\infty)}\right]\left\{N / a-Z_{a} Y_{b}-1\right\} \\
& -Z_{a} N / a=0 .
\end{aligned}
$$

This scheme for generating $Z^{(\infty)}$ can be easily randomised by randomizing the selection of $N_{i}, p_{i}$ and $q_{i}$. It may be that some of the circuits will turn out to be fractal, and others may not be so; and we are a priori unable to decisively rule on the outcome. Instead, we will give four examples which will be shown to have fractal impedances in the frequency domain, provided some specific conditions have been satisfied. These cases are:

Circuit $Z R C: \forall i \geq 1, N_{i}=N, p_{i}=a^{i}, q_{i}=1 ; \quad a>1$; $Z_{a}=R$ and $Z_{b}=[j \omega C]^{-1}$.

For this circuit, by substitution of these conditions in (6) and computing $Z^{(\infty)}$ it can be shown that the following scaling relationships in the frequency domain arise:

$$
\begin{aligned}
Z^{(\infty)}(\omega)= & R+a Z^{(\infty)}(a \omega) \\
& \cdot\left[N+a[j \omega C] Z^{(\infty)}(a \omega)\right]^{-1}, \\
Z^{(\infty)}(\omega / a)= & R+a Z^{(\infty)}(\omega) \\
& \cdot\left[N+[j \omega C] Z^{(\infty)}(\omega)\right]^{-1} .
\end{aligned}
$$

Circuit ZCR: $\forall i \geq 1, N_{i}=N, p_{i}=a^{i}, q_{i}=1 ; \quad a>1$; $Z_{a}=[j \omega C]^{-1}$ and $Z_{b}=R$.

$$
\begin{aligned}
Z^{(\infty)}(\omega)= & {[j \omega C]^{-1}+a Z^{(\infty)}(\omega / a) } \\
& \cdot\left[N+R^{-1} Z^{(\infty)}(\omega / a)\right]^{-1}, \\
a Z^{(\infty)}(a \omega)= & {[j \omega C]^{-1}+a Z^{(\infty)}(\omega) } \\
& \cdot\left[N+R^{-1} Z^{(\infty)}(\omega)\right]^{-1} .
\end{aligned}
$$

Circuit $Z R L: \forall i \geq 1, N_{i}=N, p_{i}=a^{i}, q_{i}=1 ; a>1 ;$ $Z_{a}=R$ and $Z_{b}=j \omega L$.

$$
\begin{aligned}
Z^{(\infty)}(\omega)= & R+a Z^{(\infty)}(\omega / a) \\
& \cdot\left[N+a[j \omega L]^{-1} Z^{(\infty)}(\omega / a)\right]^{-1}, \\
Z^{(\infty)}(a \omega)= & R+a Z^{(\infty)}(\omega) \\
& \cdot\left[N+[j \omega L]^{-1} Z^{(\infty)}(\omega)\right]^{-1}(10 \mathrm{~b})
\end{aligned}
$$

Circuit ZLR: $\forall i \geq 1, N_{i}=N, p_{i}=a^{i}, q_{i}=1 ; a>1 ;$ $Z_{a}=j \omega L$ and $Z_{b}=R$.

$$
\begin{aligned}
Z^{(\infty)}(\omega)= & j \omega L+Z^{(\infty)}(a \omega) \\
& \cdot\left[N+R^{-1} Z^{(\infty)}(a \omega)\right]^{-1}, \\
a Z^{(\infty)}(\omega / a)= & j \omega L+a Z^{(\infty)}(\omega) \\
& \cdot\left[N+R^{-1} Z^{(\infty)}(\omega)\right]^{-1},
\end{aligned}
$$

It should be noted that in all of these four cases, the parameter $a$ assumes a special status: as the discriminant for scale over the frequency range $\omega$. To further elucidate the self-referential character of such circuits, consider the case when $N=1$ and when $Z_{a}$ as well as $Z_{b}$ are arbitrary impedances. Then, it is possible to see that while $Z^{(0)}=Z_{a}+Z_{b}$, the higher order approximants can be set down compactly as

$$
\begin{aligned}
Z^{(i)}= & {\left[Z^{(0)} z_{1, i}+a Z_{a} Z^{(0)}+Z_{a} Z_{b}\right] } \\
& \cdot\left[a Z_{a}+Z_{b}+z_{1, i}\right]^{-1}, \quad i \geq 1,
\end{aligned}
$$

in which

$$
z_{1,1}=Z_{b}
$$

but

$$
\begin{array}{r}
z_{1, i}=Z_{b}\left[a^{2} Z_{a}+z_{2, i}\right] \cdot\left[a^{2} Z_{a}+Z_{b}+z_{2, i}\right]^{-1}, \\
i \geq 2 \cdot(13 \mathrm{~b})
\end{array}
$$

But the impedance functions $z_{2, i}, i \geq 2$, can themselves be further simplified with the help of the impedance functions $z_{3, i}, i \geq 3$, as

$$
z_{2,2}=Z_{b},
$$

but

$$
\begin{array}{r}
z_{2, i}=Z_{b}\left[a^{3} Z_{a}+z_{3, i}\right] \cdot\left[a^{3} Z_{a}+Z_{b}+z_{3, i}\right]^{-1}, \\
i \geq 3 .(14 \mathrm{~b})
\end{array}
$$

It then becomes possible to observe the self-affinity of these circuits by defining the functions

$$
\begin{aligned}
z_{i, i}= & Z_{b}, \\
z_{j, i}= & 0 ; \quad \text { if } \quad i<j, \\
z_{j, i}= & Z_{b}\left[a^{j+1} Z_{a}+z_{j+1, i}\right] \\
& \cdot\left[a^{j+1} Z_{a}+Z_{b}+z_{j+1, i}\right]^{-1} ;
\end{aligned}
$$

\section{Circuits of Infinite Size: Admittances}

The aforementioned circuits are of the "seriesparallel" form such as $Q\left(Y_{b}, Z_{a}\right)+Z_{a}$ in (4). Because of duality, it is easy to visualise "parallel-series" forms such as $Q\left(Z_{b}, Y_{a}\right)+Y_{a}$ in (5). Our building blocks here 
will be two admittances $Y_{a}=1 / Z_{a}$ and $Y_{b}=1 / Z_{b}$. In the zeroth-stage these admittances are connected in parallel; then,

$$
Y^{(0)}=Y_{a}+Y_{b}
$$

Next, $N_{1}$ branches connected in series are inserted into the branch containing $Y_{b}$, each branch of admittance $p_{1} Y_{a}+q_{1} Y_{b}$. Consquently, the admittance of the circuit at this stage can be represented by

$$
Y^{(1)}=Y_{a}+\frac{1}{Z_{b}+\frac{N_{1}}{p_{1} Y_{a}+q_{1} Y_{b}},}
$$

where $Z_{b}=1 / Y_{b}$. In the second stage, $N_{2}$ branches connected in series are inserted into the branches containing $q_{1} Y_{b}$, each branch of admittance $p_{2} Y_{a}+q_{2} Y_{b}$; hence,

$$
Y^{(2)}=Y_{a}+\frac{1}{Z_{b}+\frac{N_{1}}{p_{1} Y_{a}+\frac{1}{Z_{b} / q_{1}+\frac{N_{2}}{p_{2} Y_{a}+q_{2} Y_{b}}}}},
$$

This process also continues ad infinitum. Provided the real and the imaginary parts of the sequence $\left\{Y^{(i)}\right\}$ converge, it can be said that

$$
Y^{(\infty)}=\operatorname{Lim}_{i \rightarrow \infty} Y^{(i)}
$$

will be the admittance of the circuit so obtained. As a counterpart to (7a), if $N_{i}=N$ and $p_{i}=q_{i}=a^{i} \forall i \geq 1$, then

$$
Y^{(i)}=Y_{a}+1 /\left[Z_{b}+(N / a) Z^{(i-1)}\right] ; \quad i>0,
$$

where $Z^{(i)}=1 / Y^{(i)} \forall i \geq 0$.

The "parallel-series" counterparts of the circuits identified above as $Z R C$, etc., along their respective frequency scaling relationships, are as follows:

Circuit YRC: $\forall i \geq 1, N_{i}=N, p_{i}=a^{i}, q_{i}=1 ; \quad a>1 ;$ $Y_{a}=R^{-1}$ and $Y_{b}=j \omega C$.

$$
\begin{aligned}
Y^{(\infty)}(\omega)= & R^{-1}+a Y^{(\infty)}(\omega / a) \quad(18 \mathrm{a}) \\
& \cdot\left[N+a[j \omega C]^{-1} Y^{(\infty)}(\omega / a)\right]^{-1}, \\
Y^{(\infty)}(a \omega)= & R^{-1}+a Y^{(\infty)}(\omega) \quad(18 \mathrm{~b}) \\
& \cdot\left[N+[j \omega C]^{-1} Y^{(\infty)}(\omega)\right]^{-1} .
\end{aligned}
$$

Circuit YCR: $\forall i \geq 1, N_{i}=N, p_{i}=a^{i}, q_{i}=1 ; \quad a>1 ;$ $Y_{a}=j \omega C$ and $Y_{b}=R^{-1}$.

$$
\begin{aligned}
Y^{(\infty)}(\omega)= & j \omega C+Y^{(\infty)}(a \omega) \\
& \cdot\left[N+R Y^{(\infty)}(a \omega)\right]^{-1}, \\
a Y^{(\infty)}(\omega / a)= & j \omega C+a Y^{(\infty)}(\omega) \\
& \cdot\left[N+R Y^{(\infty)}(\omega)\right]^{-1},
\end{aligned}
$$

Circuit $Y R L: \quad \forall i \geq 1, N_{i}=N, p_{i}=a^{i}, q_{i}=1 ; a>1$; $Y_{a}=R^{-1}$ and $Y_{b}=[j \omega L]^{-1}$.

$$
\begin{aligned}
Y^{(\infty)}(\omega)= & R^{-1}+a Y^{(\infty)}(a \omega) \\
& \cdot\left[N+a[j \omega L] Y^{(\infty)}(a \omega)\right]^{-1}, \\
Y^{(\infty)}(\omega / a)= & R^{-1}+a Y^{(\infty)}(\omega) \\
& \cdot\left[N+[j \omega L] Y^{(\infty)}(\omega)\right]^{-1}, \quad(20)
\end{aligned}
$$

Circuit Y $L R: \forall i \geq 1, N_{i}=N, p_{i}=a^{i}, q_{i}=1 ; a>1$; $Y_{a}=[j \omega L]^{-1}$ and $Y_{b}=R^{-1}$.

$$
\begin{aligned}
Y^{(\infty)}(\omega)= & {[j \omega L]^{-1}+Y^{(\infty)}(\omega / a) } \\
& \cdot\left[N+R Y^{(\infty)}(\omega / a)\right]^{-1}, \\
a Y^{(\infty)}(a \omega)= & {[j \omega L]^{-1}+Y^{(\infty)}(\omega) } \\
& \cdot\left[N+R Y^{(\infty)}(\omega)\right]^{-1} .
\end{aligned}
$$

By making use of the interchanges $Z \Leftrightarrow Y, R \Leftrightarrow R^{-1}$ and $L \Leftrightarrow C$, it is easy to show that the circuits $Y R C$, $Y C R, Y R L$ and $Y L R$ are the duals, respectively, of the circuits $Z R L, Z L R, Z R C$ and $Z C R$.

\section{Discussion}

For the remainder of this paper, we will confine our discussion to the circuits $Z R C$ and $Z C R$, whose scaling behavior are, respectively, identified by $(8 \mathrm{a}, \mathrm{b})$ and $(9 \mathrm{a}, \mathrm{b})$; furthermore, we shall drop the superscript $(\infty)$ on $Z$.

First, the circuit $Z R C$ and a couple of its possible applications. Shown in Fig. 2 are the plots of the real and the imaginary parts of $Z^{(i)}$ as well as the phase angle $\varphi^{(i)}=\arctan \left[\operatorname{Im}\left\{Z^{(i)}\right\} / \operatorname{Re}\left\{Z^{(i)}\right\}\right]$ as functions of the frequency $\omega$; the building blocks are $R=1 \mathrm{k} \Omega$ and $C=1 \mathrm{nF}$. To be noted is the fact that $Z$ can be extrapolated from these graphs. It can be seen that in the low-frequency regime $(\omega R C \ll 1)$, both the real and the imaginary parts of $Z$ are proportional to $\omega^{-\zeta}$, where $\zeta=1-\ln (N) / \ln (a)=1-D, D$ being the fractal dimension [12]; indeed $Z(\omega)$ is actually proportional to $(i \omega)^{-\zeta}$. As the frequency increases, then this law is no longer followed: for the high-frequency regime $(\omega R C \gg 1$ ), the real part of $Z$ approaches the constant 
A. Lakhtakia et al. . Incommensurate Numbers, Continued Fractions, and Fractal Immittances

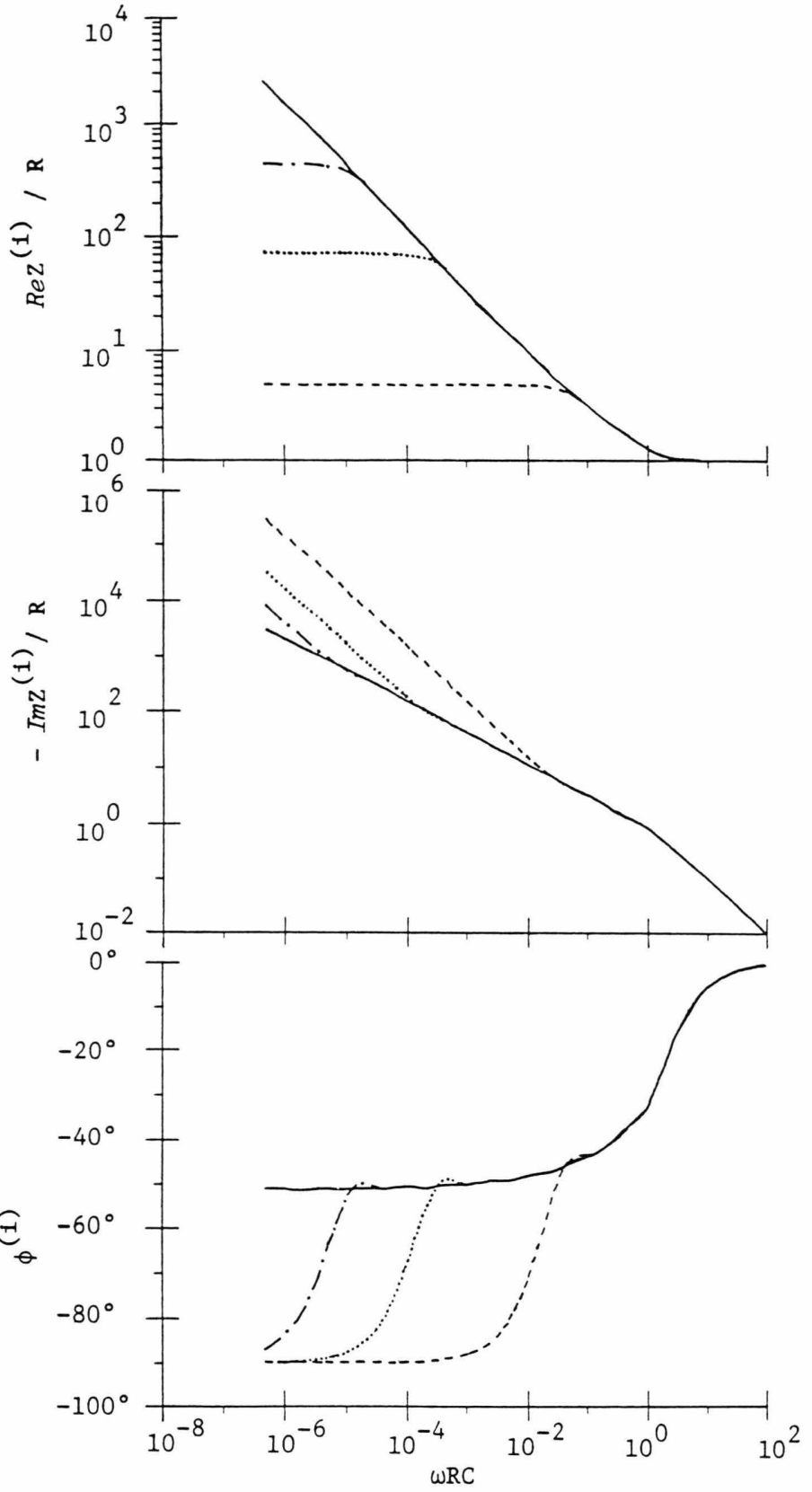

Fig. 2. Plots of $\operatorname{Re}\left\{Z^{(i)} / R\right\},-\operatorname{Im}\left\{Z^{(i)} / R\right\}$ and phase angle $\varphi^{(i)}=\arctan$ $\left[\operatorname{Im}\left\{Z^{(i)}\right\} / \operatorname{Re}\left\{Z^{(i)}\right\}\right]$ against frequency $\omega$ for the circuit $Z R C$. The building blocks $R=1 \mathrm{k} \Omega$ and $C=1 \mathrm{nF}$; the branch number $N=2$ and the branching parameter $a=5, i=2(---), 5(\cdots), 7(-\cdot-\cdot-)$ and $10(-)$. Note that $a>N^{2}$ and that for $\omega R C \ll 1, Z(\omega) \sim(i \omega)^{-\zeta}$, where $\zeta=1-\ln (N) / \ln (a)$.
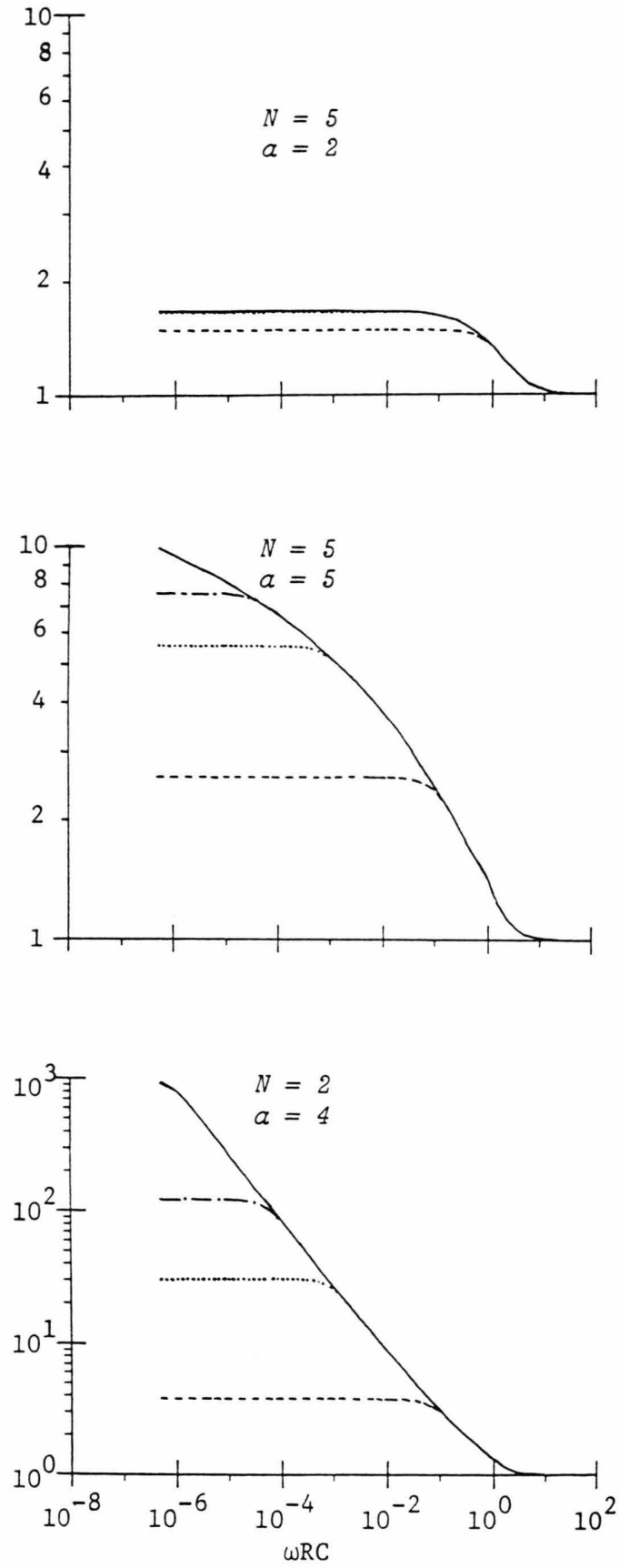

Fig. 3. Plots of $\operatorname{Re}\left\{Z^{(i)} / R\right\}$ against frequency $\omega$ for the circuit $Z R C$. The building blocks $R=1 \mathrm{k} \Omega$ and $C=1 \mathrm{nF} ; i=2(---), 5(\cdots), 7(-\cdot-\cdot-)$ and $10(-)$. Three cases have been considered: (a) $a=2, N=5$, (b) $a=N=5$, and (c) $a=4, N=2$. 
resistance $R$ and the imaginary part of $Z$ decays as $\omega^{-1}$. But the imaginary part of $Z$ is very small when compared to its real part; hence, $Z$ is almost purely resistive at high frequencies. This behavior of the $Z R C$ circuit was first noted by Liu [6] for $N=2$, and the low-frequency, constant-phase-angle response of the $Z R C$ circuit makes it a plausible model for some electrode surfaces [11,13].

It should be noted that in the calculations of Fig. 2, $a>N$. Furthermore, $\ln (N) / \ln (a)$ is a fractal dimension of the $Z R C$ circuit and lies between 0 and 0.5. Since the circuit has been used to encode in it the topography of an electrode surface, this consideration is justified [7]. From the point of view of circuit topology, however, there is no reason to confine $N<a$. In Figs. 3 and 4, the calculations of Fig. 2 are repeated for varying values of the ratio $a / N$. From these and other studies, it appears that the low-frequency, constant-phase-angle response of the circuit $Z R C$ is confined to the regime $a \geq N^{2}$. When $N \gg a$, however, another interesting characteristic appears: whereas for $\omega R C \gg 1, Z \cong R$, even in the low-frequency regime $Z$ is almost purely resistive. As the parameter $a$ is increased relative to $N^{2}$, this behavior is supplanted by the "fractal" behavior observed for $a \geq N^{2}$.

As an application of the circuit $Z R C$, we now consider the reflection coefficient $\Re(\omega)$ when a planewave is normally incident on an infinite plane [14] whose impedance can be modelled by that of the $Z R C$ circuit;

$$
\mathfrak{R}(\omega)=\left[Z(\omega)-\eta_{0}\right]\left[Z(\omega)+\eta_{0}\right]^{-1} .
$$

This reflection coefficient is plotted in Figs. 5 and 6 with $R=200 \Omega$ and $C=100 \mathrm{pF}$; the free space impedance, $\eta_{0}=120 \pi \Omega>R$. In Fig. 5 the condition $a \geq N^{2}$ has been fulfilled. For this case, it appears that in the low-frequency range, the reflection coefficient is almost equal to +1 , but in the high-frequency regime it acquires a constant negative value. Neither of these results is surprising because for $\omega R C \ll 1,|Z(\omega)| \gg \eta_{0}$, the free-space impedance; while for $\omega R C \gg 1, Z(\omega)$ $\cong R<\eta_{0}$. In other words, at low frequencies the impedance plane behaves as if it were a perfect magnetic conductor (PMC), but becomes an insulator at higher frequencies. It is to be concluded, therefore, that the $Z R C$ circuit with $a \geq N^{2}$ is a plausible model for PMC-insulator transition. When $a \ll N^{2}$, the circuit $Z R C$ also provides a model for such transitions, but somewhat of a different kind. Shown in Fig. 6 is the case when $a=2$ and $N=5$. From this figure, it is to
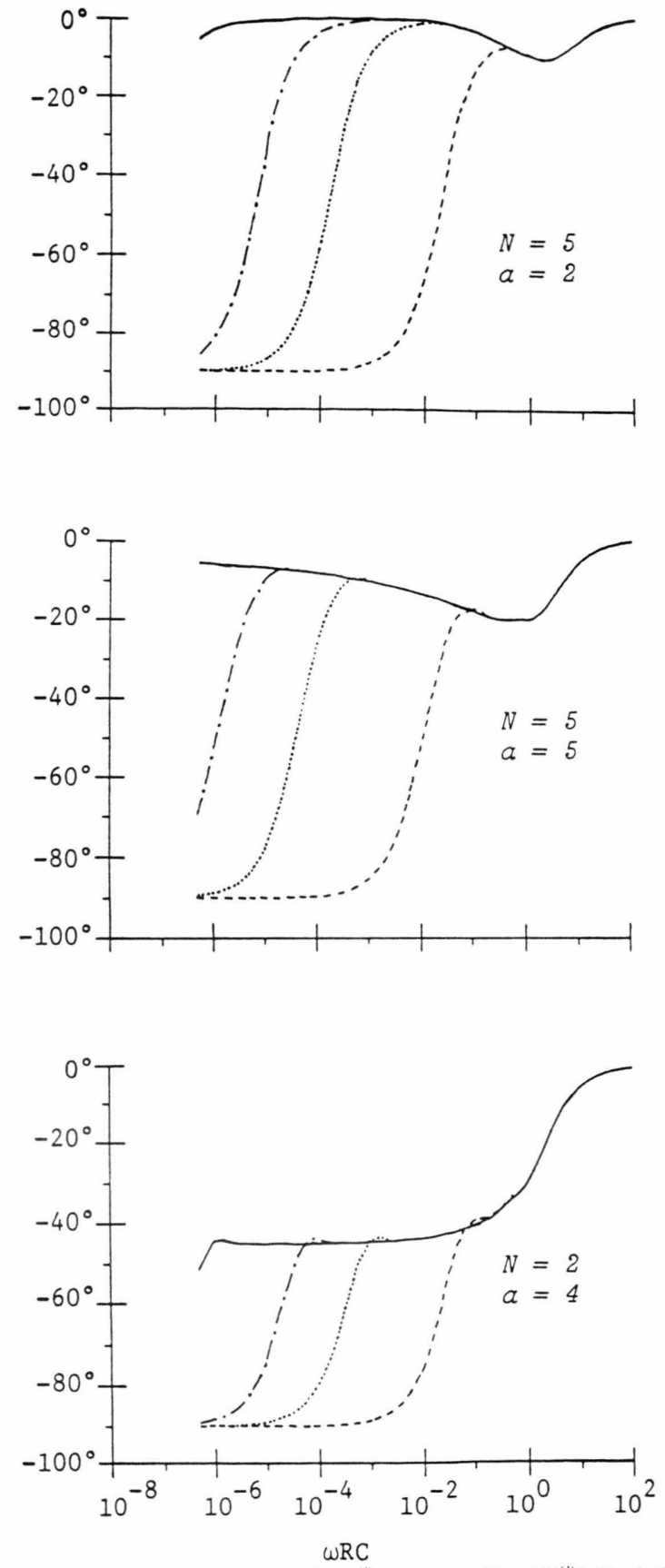

Fig. 4. Plots of phase angle $\varphi^{(i)}=\arctan \left[\operatorname{Im}\left\{\boldsymbol{Z}^{(i)}\right\} / \operatorname{Re}\left\{\boldsymbol{Z}^{(i)}\right\}\right]$ against frequency $\omega$ for the circuit $Z R C$. See Fig. 3 for other details.

be noted that both in the low- and the high-frequency regimes, $\mathfrak{R}(\omega)$ is almost always a negative real number (except for the static case when it equals unity), which is because $Z(\omega)$ is almost purely resistive in these frequency ranges. Thus, this appears to be a case of 


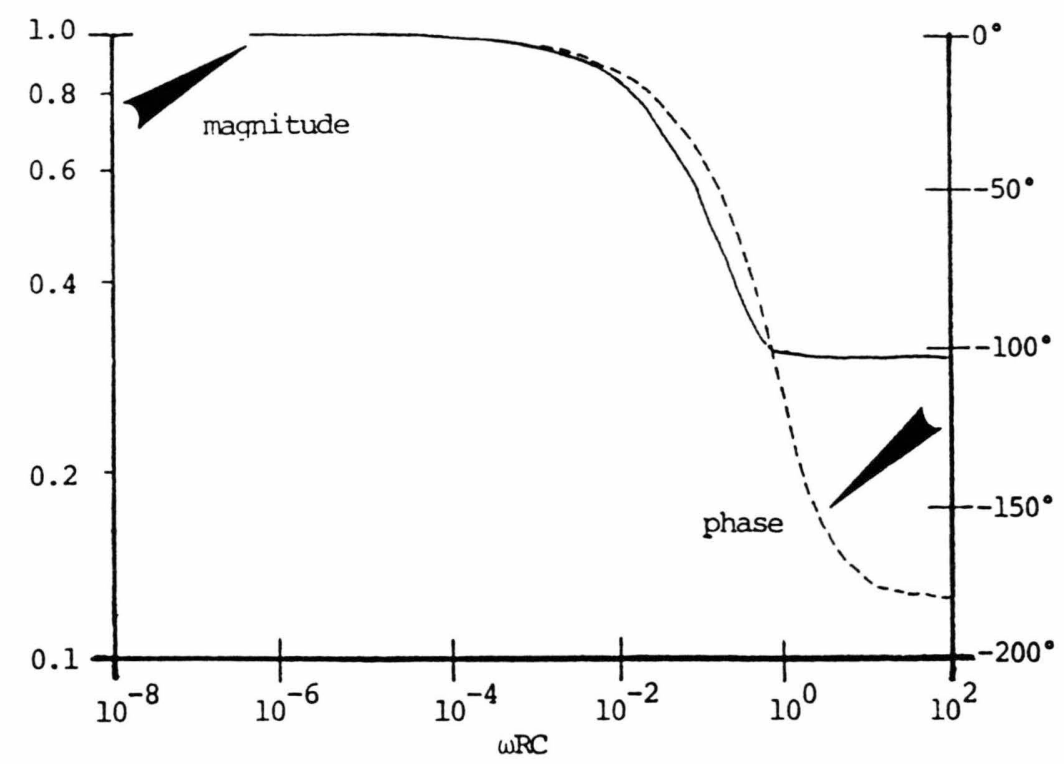

Fig. 5. Magnitude and phase of the reflection coefficient $\mathfrak{R}(\omega)$ given as a function of $\omega$ by (22). The impedance $Z(\omega)$ of the $Z R C$ circuit is approximated by $Z^{(10)}$, no difference being observed when $Z^{(7)}$ was used in place of $Z(\omega)$. The parameters $R=200 \Omega$, $C=100 \mathrm{pF}, a=5$, and $N=2$.
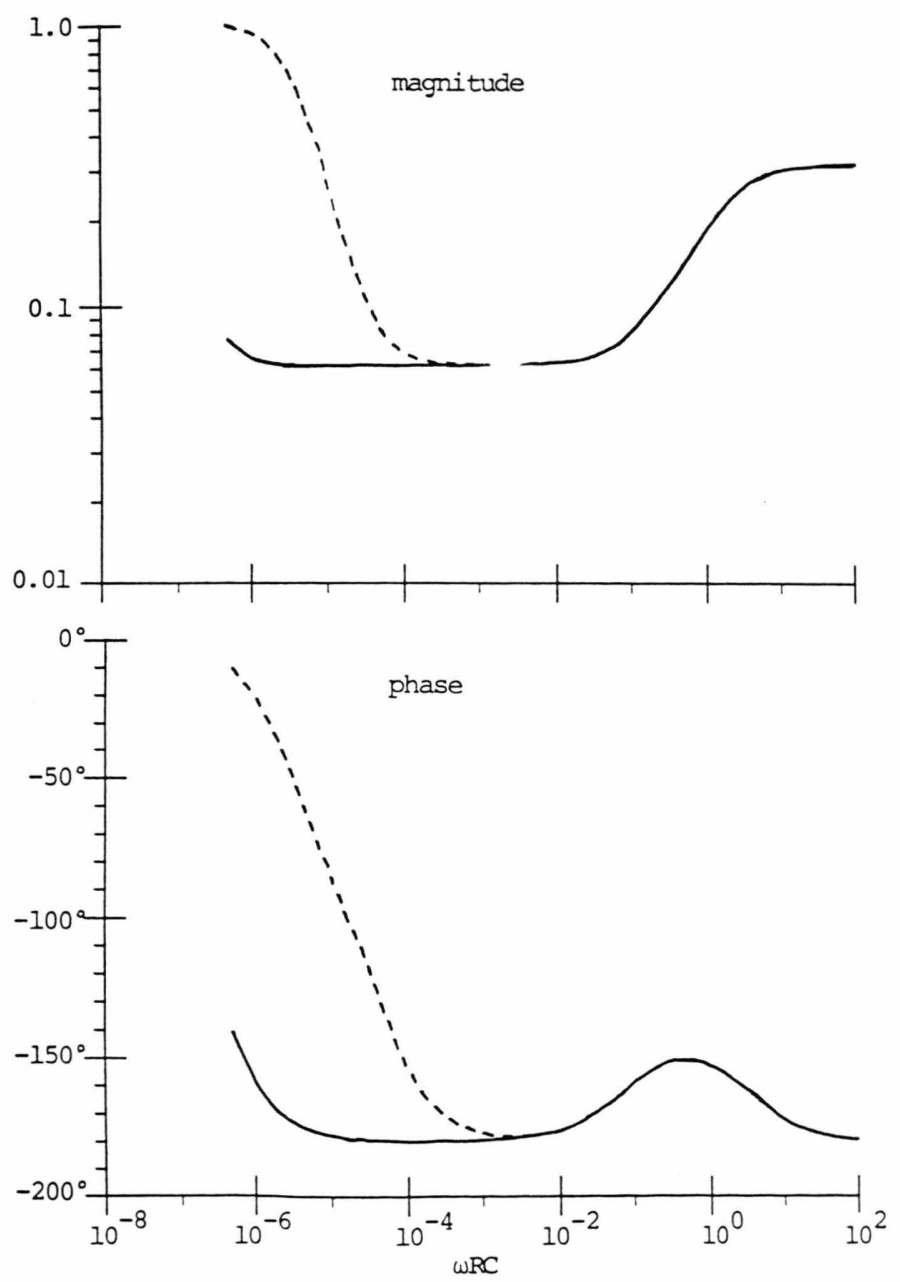

Fig. 6. Magnitude and phase of $\mathfrak{R}(\omega)$ given as a function of $\omega$ by (22). The impedance $Z(\omega)$ of the circuit $Z R C$ was approximated by $Z^{(7)}(---)$ and $Z^{(10)}(-)$. The parameters $R=200 \Omega, \quad C=$ $100 \mathrm{pF}, a=2$, and $N=5$. 

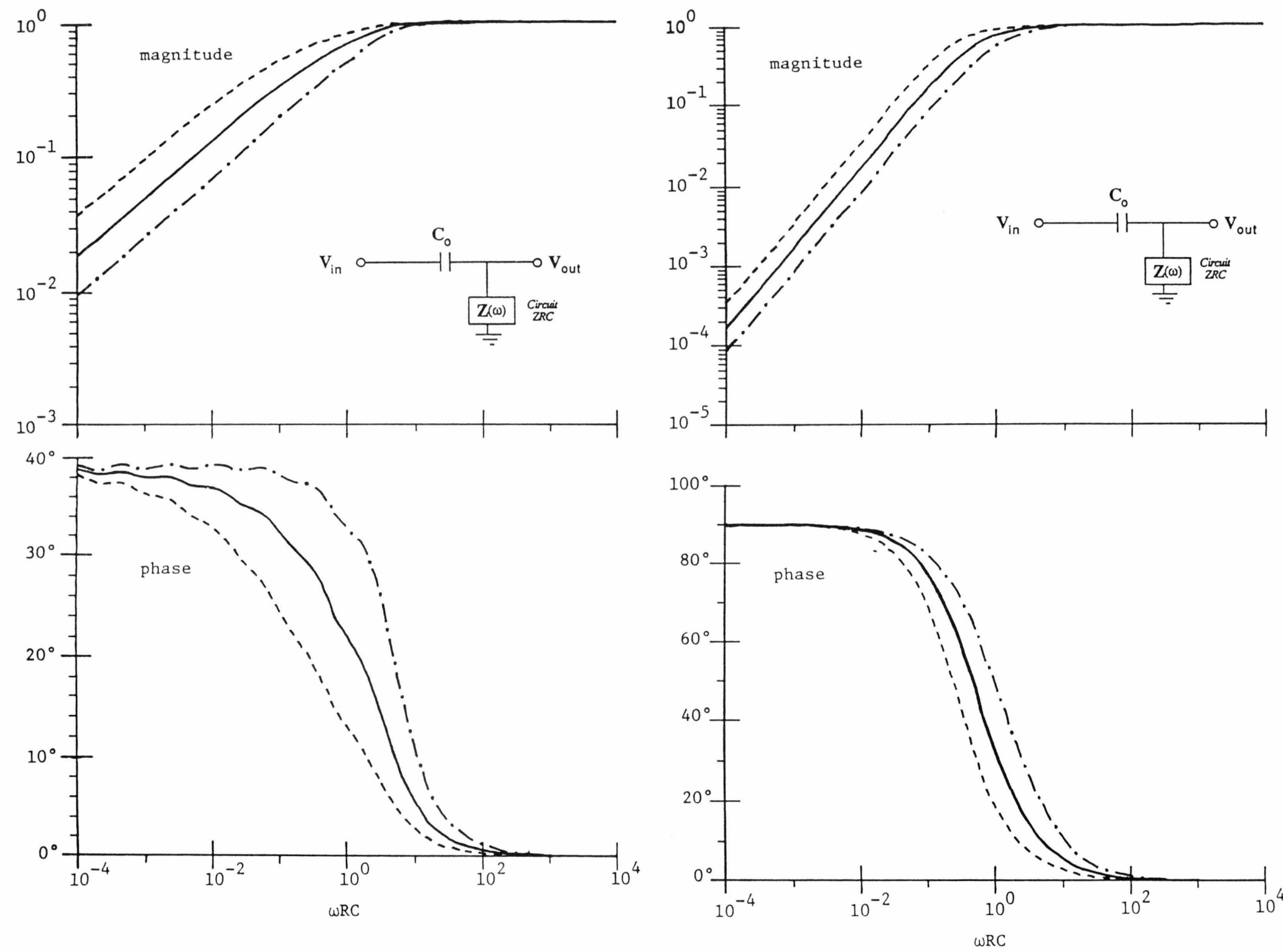

Fig. 7. Magnitude and phase of $G(\omega)$ given as a function of $\omega$ by (23). The impedance $Z(\omega)$ of the circuit $Z R C$ was approximated by $Z^{(10)}$ with $R=1 \mathrm{k} \Omega$, $C=100 \mathrm{pF}, a=5$ and $N=2$. The capacitance $C_{0}=C / 2(-\cdot \cdot-), C(-)$ and

Fig. 8. Same as Fig. 7, except $a=2$ and $N=5$. 

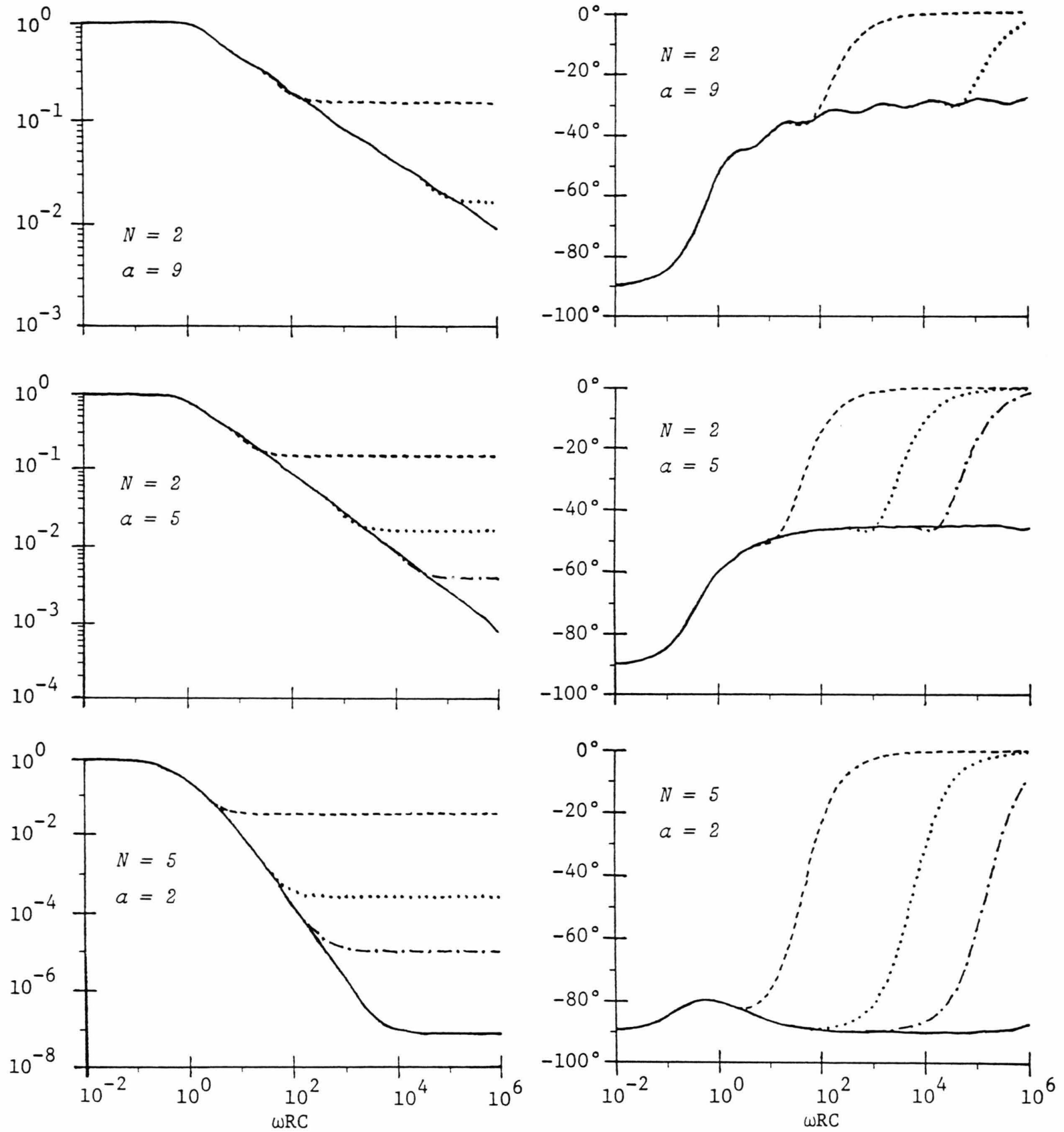

Fig. 9. Plots of $\operatorname{Re}\left\{Z^{(i)} / R\right\}$ against frequency $\omega$ for the circuit $Z C R$. The building blocks $R=1 \mathrm{k} \Omega$ and $C=1 \mathrm{nF}$; $i=2(---), 5(\cdots), 7(-\cdot-\cdot-)$ and $10(-)$. Three cases have been considered: (a) $a=9, N=2$, (b) $a=5, N=2$, and (c)

Fig. 10. Plots of phase angle $\varphi^{(i)}=\arctan \left[\operatorname{Im}\left\{Z^{(i)}\right\} / \operatorname{Re}\left\{Z^{(i)}\right\}\right]$ against frequency $\omega$ for the circuit $Z C R$. See Fig. 9 for other details. $a=2, N=5$. 
insulator-insulator transition, perhaps typifying a curious (relaxation?) mechanism in which the dielectric constant changes from a low value to a higher value as the frequency increases $[15,16]$. Parenthetically, it should be noted that this second case can also be interpreted to be symptomatic of a relaxation in which the (magnetic) permeability changes from a high value to a lower one with the increase in frequency [17]. Both of these impedance plane models merit further attention. It must also be noted that the scattering plane need not be planar: it is possible to think of a rough, bimaterial interface as a perfectly smooth plane possessed with a space-dependent impedance [18]. This impedance is not only a function of the geometry of the actual (rough) surface, but also of the frequency $\omega$ and the field incident on the surface.

Finally, the circuit $Z R C$ may be used as part of a filter, as shown in the insets of Figs. 7 and 8. For these two figures, the open-circuit voltage gain was computed via

$$
G(\omega)=Z(\omega)\left[Z(\omega)+\left(j \omega C_{0}\right)^{-1}\right],
$$

in which $Z(\omega)$ was approximated by $Z^{(10)}(\omega)$, $R=1 \mathrm{k} \Omega$ and $C=100 \mathrm{pF}$. The capacitance $C_{0}$ was assumed to have three values: $C_{0}=C / 2, C$ and $2 C$. In Fig. 7, $a=5$ and $N=2$, so that $a>N^{2}$; and in the latter figure, $a=2$ and $N=5$ so that $a \ll N^{2}$. It is to be noted that for either case, $G(\omega) \cong 1$ when $\omega R C \gg 1$, so that the high frequencies are passed on by the filters without any distortion. The low frequencies $(\omega R C \ll 1)$ are, however, attenuated, so that these filters are essentially high pass; furthermore, the rolloff appears to be rather insensitive to the capacitance ratio $C_{0} / C$. But the roll-off in Fig. 7 is about $8.4 \mathrm{~dB} /$ decade, and in Fig. 8 it is about $20 \mathrm{~dB} /$ decade, which suggests that as an element of these filters, the circuit $Z R C$ with $a \ll N^{2}$ is certainly the more efficient one. This is because, as evinced by Fig. 3, the circuit $Z R C$ with $a \ll N^{2}$ behaves much like a pure resistance in both the low- and the high-frequency ranges.

Just as the response of the circuit $Z R C$ is dominated by its real (resistive) part, the circuit $Z C R$ is dominated by its imaginary (capacitative) part. Shown in Figs. 9 and 10 are the plots of the real part and the
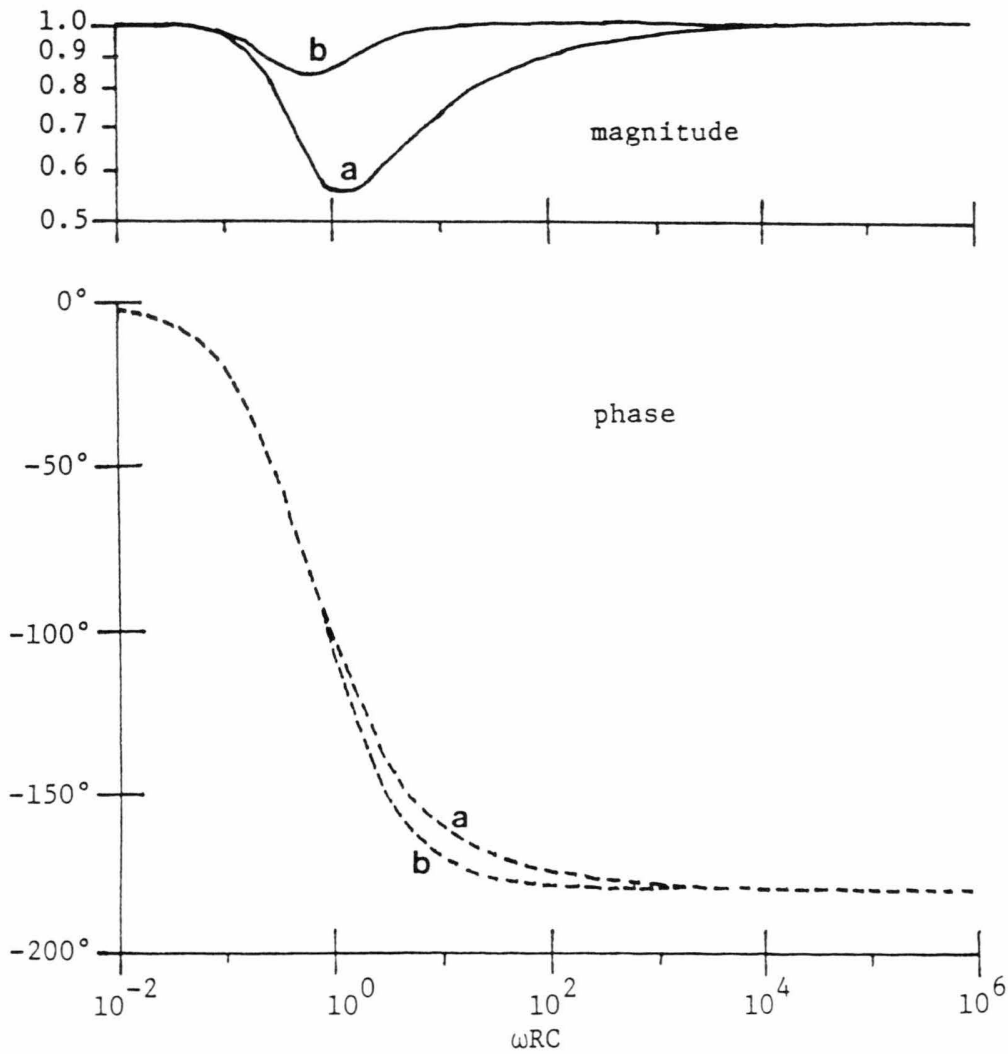

Fig. 11. Magnitude and phase of the reflection coefficient $\mathfrak{R}(\omega)$ given as a function of $\omega$ by (22). The impedance $Z(\omega)$ of the $Z C R$ circuit is approximated by $Z^{(10)}$, no difference being observed when $Z^{(7)}$ was used in place of $Z(\omega) ; \quad R=200 \Omega, \quad C=100 \mathrm{pF}$. The branching parameters are (a) $a=5$, $N=2$, and (b) $a=2, N=5$. 

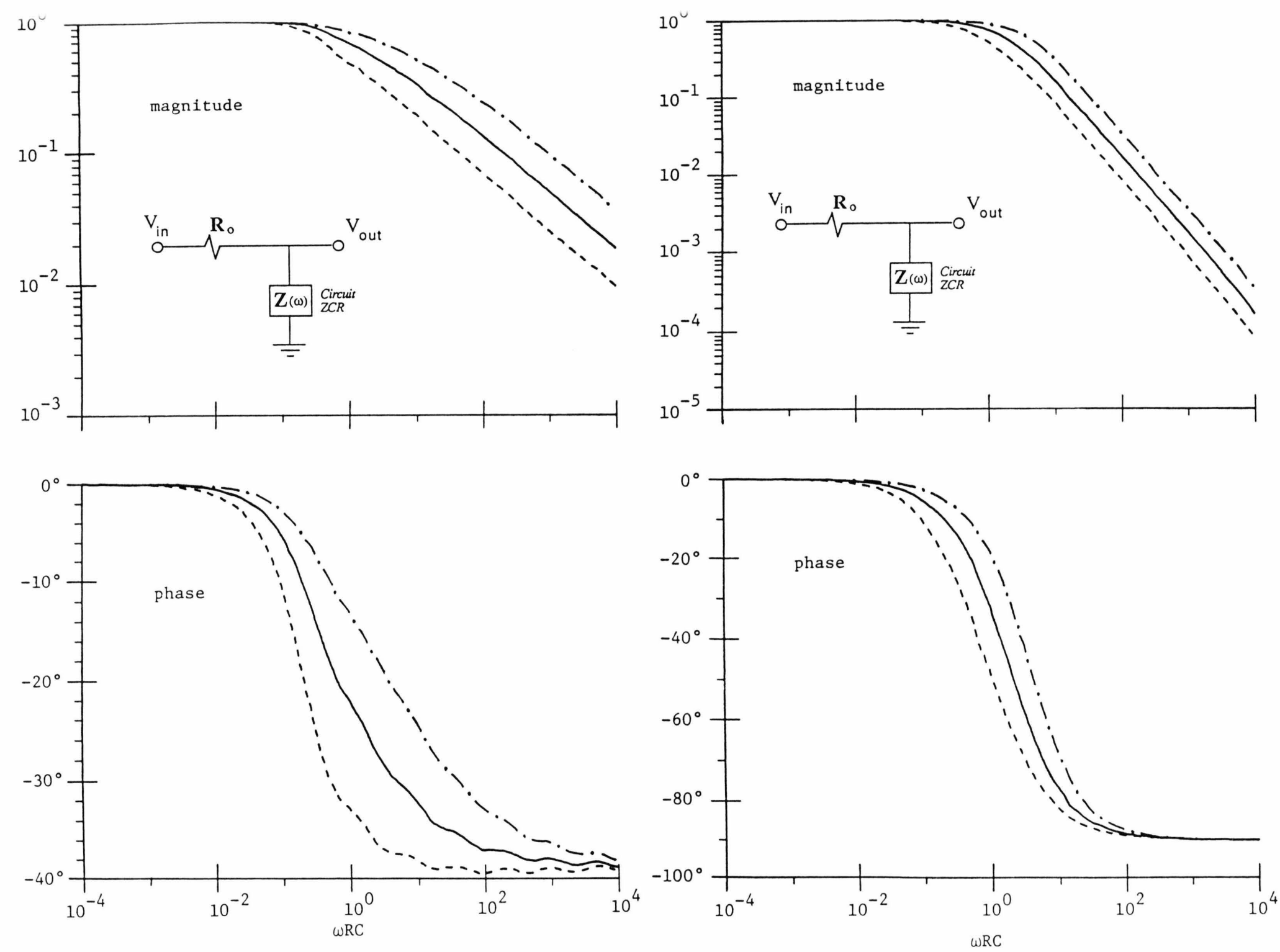

Fig. 12. Magnitude and phase of $G(\omega)$ given as a function of $\omega$ by (23). The impedance $Z(\omega)$ of the circuit $Z C R$ was approximated by $Z^{(10)}$ with $R=1 \mathrm{k} \Omega$, $C=100 \mathrm{pF}, a=5$ and $N=2$. The resistance $R_{0}=R / 2(-\cdot \cdot \cdot), R(-)$ and

Fig. 13. Same as Fig. 12, except $a=2$ and $N=5$. 
phase, respectively, of the approximants $Z^{(i)}$ for some circuits $Z C R$; the building blocks are $R=1 \mathrm{k} \Omega$ and $C=1 \mathrm{nF}$. To be noted is the fact that in the static limit $\omega \rightarrow \infty, Z(\omega)$ is almost purely capacitative. Furthermore, $\operatorname{Re} Z(\omega)=R$ at low frequencies $(\omega R C \ll 1)$, but for $\omega R C \gg 1$, it appears that $\operatorname{Re} Z(\omega)$ is proportional to $\omega^{-\zeta}$. When $a \geq N^{2}$, then $\zeta=\ln (N) / \ln (a)$, which is a number less than 0.5 ; as the ratio $N / a$ increases, however, $\zeta$ does so too and achieves values higher than unity. For $a \geq N^{2}$, the impedance of the circuit $Z C R$ also scales as $(i \omega)^{-\zeta}$ for $\omega R C \gg 1$, but this relationship becomes slightly imprecise in its phase when $a \gg N^{2}$. On the other hand, when $a \ll N^{2}$, then both in the low and the high frequency limits, $Z(\omega)$ is almost purely capacitative.

Using (22), the reflection coefficient $\mathfrak{R}(\omega)$ is computed in Fig. 11 for a planewave normally incident on an infinite plane whose impedance is modelled by the circuit $Z C R ; R=200 \Omega$ and $C=100 \mathrm{pF}$. It is apparent from this figure that, qualitatively speaking, there is no difference between the cases (a) $a \geq N^{2}$ and (b) $a \ll N^{2}$. In the low frequency range, $\Re(\omega)$ tends towards +1 ; and in the high frequency regime, $\mathfrak{R}(\omega)$ is approximately equal to negative unity. Both of these observations are consistent with the capacitative nature of the circuit ZCR. Also, it appears that the impedance plane behaves as a perfect electric conductor (PEC) for $\omega R C \gg 1$, so that the circuit $Z C R$ models PMC-PEC transitions, regardless of the specific values of the branching parameters $a$ and $N$. Pertinent to the scattering of acoustic waves, this can also be interpreted as a transition from an acoustically hard surface to an acoustically soft one.

The open-circuit voltage gain $G(\omega)$ of the filter, shown in the insets of Figs. 12 and 13, has been computed via the relationship (23). For these calculations, $Z(\omega)$ of the circuit $Z C R$ was approximated by $Z^{(10)}(\omega)$, $R=1 \mathrm{k} \Omega$ and $C=100 \mathrm{pF}$. The resistance $R_{0}$ was assumed to have three values: $R_{0}=R / 2, R$ and $2 R$. In Fig. 12, $a=5$ and $N=2$, so that $a>N^{2}$; while in Fig. 13, $a \ll N^{2}$ and $a=2$ with $N=5$. It is to be noted that for either case, $G(\omega) \cong 1$ when $\omega R C \ll 1$ : this implies that the low frequencies are passed on by the filters without any significant distortion. On the other hand, the high frequencies $(\omega R C \gg 1)$ are attenuated, the attenuation being rather insensitive to the ratio $R_{0} / R$. But this roll-off rate in Fig. 12 is about $8.5 \mathrm{~dB} /$ decade, while in Fig. 13 it is almost $20 \mathrm{~dB} /$ decade. This suggests that the use of the circuit $Z C R$ with $a \ll N^{2}$ is certainly more efficient for these low-pass filters.
There are several envisioned applications of this mathematical formalism to the modelling of real thin film materials. First, it has already been shown that the $Z R C$ circuit is a plausible model for the interfacial impedances of rough electrode surfaces in electrochemical cells [6].

Second, cermet films are mixtures of metallic and insulating materials which form a fractal [19-21] sponge-like connected network of either the metallic or the insulator phase, depending upon the concentration of the metal. A test for whether such materials are analogous would be to measure the reflection coefficient of thin $(\sim 100-500 \AA)$ cermet films when a planewave is normally incident and compare the results with applicable formulas [14] similar to (22). The use of the $Z R C$ and the $Z C R$ circuit impedances to simulate the impedance of an infinite plane should turn out to be analogous to the metallic and the insulating cermet states [20], respectively.

Lastly, for thicker films $(1-100 \mu)$ of essentially a single-phase material prepared under conditions of low adatom mobility, it has been demonstrated that the top surface is cauliflower-like and is directly related to a conical void network structure [22]. Although these void networks appear fractal, there has been no quantitative description of them [23]. The present work suggests ways to test and measure the fractal nature of such films. The impedances of the void regions and that of the "bulk" conical regions are certainly different; the film properties should have analogy to both the $Z R C$ (ionic diffusion and electrical conductivity) as well as the $Z C R$ (permittivity) circuits.

\section{Acknowledgement}

This work was supported in part by the United States Air Force Office of Scientific Research.

\section{Appendix: Generalized Continued Fractions}

Rigorously speaking, a continued fraction should be defined [24] through an ordered set of numbers $\left\{a_{n}\right\},\left\{b_{n}\right\}$ and $\left\{\zeta_{n}\right\}$, where $a_{0}, a_{1}, a_{2}, \ldots$ and $b_{1}, b_{2}, \ldots$ are complex numbers with all $b_{n} \neq 0$, and where $\left\{\zeta_{n}\right\}$ is a sequence in the extended complex plane. The numbers $\zeta_{n}$ approximate the continued fraction $z$, and assuming that the continued fraction is infinitely long,

$$
z=\operatorname{Lim}_{n \rightarrow \infty} \zeta_{n}
$$


where

$$
\zeta_{n}=a_{0}+\frac{b_{1}}{a_{1}+\frac{b_{2}}{a_{2}+\ldots}+\ldots+b_{n} / a_{n}} .
$$

The convergence (A1) is subject to some conditions, which have been discussed in detail in Chaps. 3 and 4 of the book by Jones and Thron [24]. It will suffice for the present purposes to indicate here that the sequence $\left\{\zeta_{n}\right\}$ does converge to $z$, provided

$$
\left|a_{n}\right| \geq\left|b_{n}\right|+1 ; \quad n=1,2,3, \ldots
$$

[1] P. M. Chirlian, Basic Network Theory, McGraw-Hill, New York, 1969, p. 494

[2] M. Ya. Azbel, Phys. Rev. Lett. 43, 1954 (1979).

[3] F. D. M. Haldane, Phys. Rev. Lett. 51, 605 (1983).

[4] K. A. Chao, J. Phys. A 19, 2907 (1986).

[5] K. Ikeda, M. Mizuno, Phys. Rev. Lett. 53, 1340 (1984).

[6] S. H. Liu, Phys. Rev. Lett. 55, 529 (1985).

[7] T. Kaplan, S. H. Liu, and L. J. Gray, Phys. Rev. B 34, 4870 (1986).

[8] M. R. Schroeder, Number Theory in Science and Communication, Springer-Verlag, Berlin, Chapter 5 (1986).

[9] N. H. McCoy, The Theory of Numbers, MacMillan, New York, p. 99 (1971).

[10] C. D. Olds, Continued Fractions, Math. Assoc. America, p. 56 (1963)

[11] P. H. Bottelberghs and G. H. J. Broers, J. Electroanal. Chem. 67, 155 (1976).

[12] A. Lakhtakia, R. Messier, V. V. Varadan, and V. K. Varadan, Phys. Lett. A 118, 54 (1986).

[13] J. R. MacDonald, J. Electroanal. Chem. 223, 25, (1987).

[14] J. D. Kraus, Electromagnetics, McGraw-Hill, New York 1984, Sect. $10-16$.
For the sake of computational tractability, it is convenient to express the $n$th approximant as

$$
\zeta_{n}=B_{n} / A_{n}
$$

where

$$
\begin{array}{ll}
B_{-1}=A_{0}=1, \quad B_{0}=a_{0}, & A_{-1}=0, \\
A_{n}=a_{n} A_{n-1}+b_{n} A_{n-2}, & n=1,2,3 \ldots, \\
B_{n}=a_{n} B_{n-1}+b_{n} B_{n-2}, & n=1,2,3 \ldots .
\end{array}
$$

[15] V. K. Farkya and D. Kumar, Ind. J. Pure Appl. Phys. 24, 592 (1986).

[16] A. Ramsamugh and F. Brouers, Phil. Mag. Lett. 55, 301 (1987).

[17] R. Barrue, J. C. Perron, and J. Robert, Rev. Phys. Appl. (Paris) 22, 399 (1987)

[18] A. Hessel and A. A. Oliner, Appl. Opt. 4, 1275 (1965).

[19] A. Kapitulnik and G. Deutscher, Phys. Rev. Lett. 49, 1444 (1982).

[20] R. B. Laibowitz, E. I. Allessandrini, and G. Deutscher, Phys. Rev. B 25, 2965 (1982).

[21] R. F. Voss, R. B. Laibowitz, and E. I. Allessandrini, Phys. Rev. Lett. 49, 1441 (1982).

[22] R. Messier and J. E. Yehoda, J. Appl. Phys. 58, 3739 (1985).

[23] R. Messier, J. Vac. Sci. Technol. A 4, 490 (1986).

[24] W. B. Jones and W. J. Thron, Continued Fractions: Analytic Theory and Applications, Addison-Wesley, Reading, Mass. 1980. 